\title{
A conjugate gradient algorithm for large-scale unconstrained optimization problems and nonlinear equations
}

\author{
Gonglin Yuan ${ }^{1}$ and Wujie $\mathrm{Hu}^{1 *}$ (D)
}

"Correspondence: hwj@gxu.edu.cn 'College of Mathematics and Information Science, Guangx University, Nanning, P.R. China

\begin{abstract}
For large-scale unconstrained optimization problems and nonlinear equations, we propose a new three-term conjugate gradient algorithm under the Yuan-Wei-Lu line search technique. It combines the steepest descent method with the famous conjugate gradient algorithm, which utilizes both the relevant function trait and the current point feature. It possesses the following properties: (i) the search direction has a sufficient descent feature and a trust region trait, and (ii) the proposed algorithm globally converges. Numerical results prove that the proposed algorithm is perfect compared with other similar optimization algorithms.
\end{abstract}

MSC: $90 C 26$

Keywords: Conjugate gradient; Descent property; Global convergence

\section{Introduction}

It is well known that the model of small- and medium-scale smooth functions is simple since it has many optimization algorithms, such as Newton, quasi-Newton, and bundle algorithms. Note that three algorithms fail to effectively address large-scale optimization problems because they need to store and calculate relevant matrices, whereas the conjugate gradient algorithm is successful because of its simplicity and efficiency.

The optimization model is an important mathematic problem since it has been applied to various fields such as economics, engineering, and physics (see [1-12]). Fletcher and Reeves [13] successfully address large-scale unconstrained optimization problems on the basis of the conjugate gradient algorithm and obtained amazing achievements. The conjugate gradient algorithm is increasingly famous because of its simplicity and low requirement of calculation machine. In general, a good conjugate gradient algorithm optimization algorithm includes a good conjugate gradient direction and an inexact line search technique (see [14-18]). At present, the conjugate gradient algorithm is mostly applied to smooth optimization problems, and thus, in this paper, we propose a modified LS conjugate gradient algorithm to solve large-scale nonlinear equations and smooth problems. The common algorithms of addressing nonlinear equations include Newton and quasiNewton methods (see [19-21]), gradient-based, CG methods (see [22-24]), trust region methods (see [25-27]), and derivative-free methods (see [28]), and all of them fail to address large-scale problems. The famous optimization algorithms of spectral gradient

(c) The Author(s) 2018. This article is distributed under the terms of the Creative Commons Attribution 4.0 International License (http://creativecommons.org/licenses/by/4.0/), which permits unrestricted use, distribution, and reproduction in any medium, provided you give appropriate credit to the original author(s) and the source, provide a link to the Creative Commons license, and indicate if changes were made. 
approach, limited-memory quasi-Newton method and conjugate gradient algorithm, are suitable to solve large-scale problems. $\mathrm{Li}$ and $\mathrm{Li}$ [29] proposed various algorithms on the basis of modified PRP conjugate gradient, which successfully solve large-scale nonlinear equations.

A famous mathematic model is given by

$$
\min \left\{f(x) \mid x \in \Re^{n}\right\},
$$

where $f: \Re^{n} \rightarrow \Re$ and $f \in C^{2}$. The relevant model is widely used in life and production. However, it is a complex mathematic model since it needs to meet various conditions in the field [30-33]. Experts and scholars have conducted numerous in-depth studies and have made some significant achievements (see [14, 34, 35]). It is well known that the steepest descent algorithm is perfect since it is simple and its computational and memory requirements are low. It is regrettable that the steepest descent method sometimes fails to solve problems due to the "sawtooth phenomenon". To overcome this flaw, experts and scholars presented an efficient conjugate gradient method, which provides high performance with a simple form. In general, the mathematical formula for (1.1) is

$$
x_{k+1}=x_{k}+\alpha_{k} d_{k}, \quad k \in\{0,1,2, \ldots\}
$$

where $x_{k+1}$ is the next iteration point, $\alpha_{k}$ is the step length, and $d_{k}$ is the search direction. The famous weak Wolfe-Powell (WWP) line search technique is determined by

$$
g\left(x_{k}+\alpha_{k} d_{k}\right)^{T} d_{k} \geq \rho g_{k}^{T} d_{k}
$$

and

$$
f\left(x_{k}+\alpha_{k} d_{k}\right) \leq f_{k}+\varphi \alpha_{k} g_{k}^{T} d_{k}
$$

where $\varphi \in(0,1 / 2), \alpha_{k}>0$, and $\rho \in(\varphi, 1)$. The direction $d_{k+1}$ is often defined by the formula

$$
d_{k+1}= \begin{cases}-g_{k+1}+\beta_{k} d_{k} & \text { if } k \geq 1, \\ -g_{k+1} & \text { if } k=0,\end{cases}
$$

where $\beta_{k} \in \mathfrak{R}$. An increasing number of efficient conjugate gradient algorithms have been proposed by different expressions of $\beta_{k}$ and $d_{k}$ (see [13, 36-42] etc.). The well-known PRP algorithm is given by

$$
\beta_{k}^{\mathrm{PRP}}=\frac{g_{k+1}^{T}\left(g_{k+1}-g_{k}\right)}{\left\|g_{k}\right\|\left\|g_{k}\right\|}
$$

where $g_{k}, g_{k+1}$, and $f_{k}$ denote $g\left(x_{k}\right), g\left(x_{k+1}\right)$, and $f\left(x_{k}\right)$, respectively; $g_{k+1}=g\left(x_{k+1}\right)=\nabla f\left(x_{k+1}\right)$ is the gradient function at the point $x_{k+1}$. It is well known that the PRP algorithm is efficient but has shortcomings, as it does not possess global convergence under the WWP line search technique. To solve this complex problem, Yuan, Wei, and Lu [43] developed 
the following creative formula (YWL) for the normal WWP line search technique and obtained many fruitful theories:

$$
f\left(x_{k}+\alpha_{k} d_{k}\right) \leq f\left(x_{k}\right)+\iota \alpha_{k} g_{k}^{T} d_{k}+\alpha_{k} \min \left[-\iota_{1} g_{k}^{T} d_{k}, \iota \alpha_{k}\left\|d_{k}\right\|^{2} / 2\right]
$$

and

$$
g\left(x_{k}+\alpha_{k} d_{k}\right)^{T} d_{k} \geq \tau g_{k}^{T} d_{k}+\min \left[-\iota_{1} g_{k}^{T} d_{k}, \iota \alpha_{k}\left\|d_{k}\right\|^{2}\right],
$$

where $\iota \in\left(0, \frac{1}{2}\right), \alpha_{k}>0, \iota_{1} \in(0, \iota)$, and $\tau \in(\iota, 1)$. Further work can be found in [24]. Based on the innovation of YWL line search technique, Yuan pay much attention to normal Armijo line search technique and make further study. They proposed an efficient modified Armijo line search technique:

$$
f\left(x_{k}+\alpha_{k} d_{k}\right) \leq f\left(x_{k}\right)+\lambda \alpha_{k} g_{k}^{T} d_{k}+\alpha_{k} \min \left[-\lambda_{1} g_{k}^{T} d_{k}, \lambda \frac{\alpha_{k}}{2}\left\|d_{k}\right\|^{2}\right]
$$

where $\lambda, \gamma \in(0,1), \lambda_{1} \in(0, \lambda)$, and $\alpha_{k}$ is the largest number of $\left\{\gamma^{k} \mid k=0,1,2, \ldots\right\}$. In addition, experts and scholars pay much attention to the three-term conjugate gradient formula. Zhang et al. [44] proposed the famous formula

$$
d_{k+1}=-g_{k+1}+\frac{g_{k+1}^{T} y_{k} d_{k}-d_{k}^{T} g_{k+1} y_{k}}{g_{k}^{T} g_{k}} .
$$

Nazareth [45] proposed the new formula

$$
d_{k+1}=-y_{k}+\frac{y_{k}^{T} y_{k}}{y_{k}^{T} d_{k}} d_{k}+\frac{y_{k-1}^{T} y_{k}}{y_{k-1}^{T} d_{k-1}} d_{k-1}
$$

where $y_{k}=g_{k+1}-g_{k}$ and $s_{k}=x_{k+1}-x_{k}$. These two conjugate gradient methods have a sufficient descent property but fail to have the trust region feature. To improve these methods, Yuan et al. [46, 47] make a further study and get some good results. This inspires us to continue the study and extend the conjugate gradient methods to get better results. In this paper, motivated by in-depth discussions, we express a modified conjugate gradient algorithm, which has the following properties:

- The search direction has a sufficient descent feature and a trust region trait.

- Under mild assumptions, the proposed algorithm possesses the global convergence.

- The new algorithm combines the steepest descent method with the conjugate gradient algorithm.

- Numerical results prove that it is perfect compared to other similar algorithms.

The rest of the paper is organized as follows. The next section presents the necessary properties of the proposed algorithm. The global convergence is stated in Sect. 3. In Sect. 4, we report the corresponding numerical results. In Sect. 5, we introduce the large-scale nonlinear equations and express the new algorithm. Some necessary properties are listed in Sect. 6. The numerical results are reported in Sect. 7. Without loss of generality, $f\left(x_{k}\right)$ and $f\left(x_{k+1}\right)$ are replaced by $f_{k}$ and $f_{k+1}$, and $\|\cdot\|$ is the Euclidean norm. 


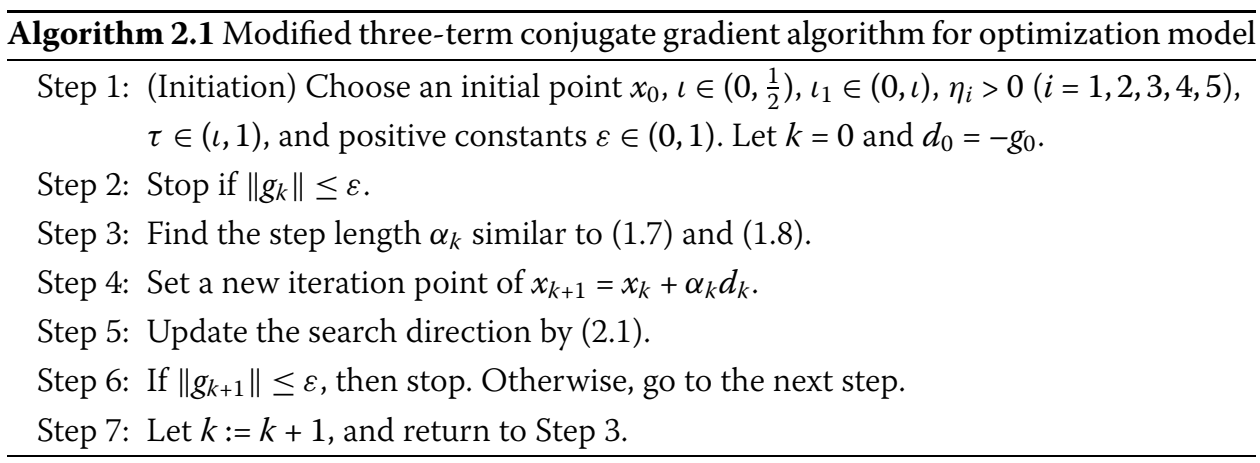

\section{New modified conjugate gradient algorithm}

Experts and scholars have conducted thorough research on the conjugate gradient algorithm and have obtained rich theoretical achievements. In light of the previous work by experts on the conjugate gradient algorithm, a sufficient descent feature is necessary for the global convergence. Thus, we express a new conjugate gradient algorithm under the YWL line search technique as follows:

$$
d_{k+1}= \begin{cases}-\eta_{1} g_{k+1}+\left(1-\eta_{1}\right)\left(d_{k}^{T} g_{k+1} y_{k}^{*}-g_{k+1}^{T} y_{k}^{*} d_{k}\right) / \delta & \text { if } k \geq 1, \\ -g_{k+1} & \text { if } k=0\end{cases}
$$

where $\delta=\max \left(\min \left(\eta_{5}\left|s_{k}^{T} y_{k}^{*}\right|,\left|d_{k}^{T} y_{k}^{*}\right|\right), \eta_{2}\left\|y_{k}^{*}\right\|\left\|d_{k}\right\|, \eta_{3}\left\|g_{k}\right\|^{2}\right)+\eta_{4} *\left\|d_{k}\right\|^{2}, y_{k}^{*}=g_{k+1}-\frac{\left\|g_{k+1}\right\|^{2}}{\left\|g_{k}\right\|^{2}} g_{k}$, and $\eta_{i}>0(i=1,2,3,4,5)$. The search direction is well defined, and its properties are stated in the next section. Now, we introduce a new conjugate gradient algorithm called Algorithm 2.1.

\section{Important characteristics}

This section lists some important properties of sufficient descent, the trust region, and the global convergence of Algorithm 2.1. It expresses the necessary proof.

Lemma 3.1 If search direction $d_{k}$ meets condition of (2.1), then

$$
g_{k}^{T} d_{k}=-\eta_{1}\left\|g_{k}\right\|^{2}
$$

and

$$
\left\|d_{k}\right\| \leq\left(\eta_{1}+2\left(1-\eta_{1}\right) / \eta_{2}\right)\left\|g_{k}\right\|
$$

Proof It is obvious that formulas of (3.1) and (3.2) are true for $k=0$.

Now consider the condition $k \geq 1$. Similarly to (2.1), we have

$$
\begin{aligned}
g_{k+1}^{T} d_{k+1} & =g_{k+1}^{T}\left[-\eta_{1} g_{k+1}+\left(1-\eta_{1}\right)\left(d_{k}^{T} g_{k+1} y_{k}^{*}-g_{k+1}^{T} y_{k}^{*} d_{k}\right) / \delta\right] \\
& =-\eta_{1}\left\|g_{k+1}\right\|^{2}+\left(1-\eta_{1}\right)\left(g_{k+1}^{T} d_{k}^{T} g_{k+1} y_{k}^{*}-g_{k+1}^{T} g_{k+1}^{T} y_{k}^{*} d_{k}\right) / \delta \\
& =-\eta_{1}\left\|g_{k+1}\right\|^{2}
\end{aligned}
$$


and

$$
\begin{aligned}
\left\|d_{k+1}\right\| & =\left\|-\eta_{1} g_{k+1}+\left(1-\eta_{1}\right)\left(d_{k}^{T} g_{k+1} y_{k}^{*}-g_{k+1}^{T} y_{k}^{*} d_{k}\right) / \delta\right\| \\
& \leq \eta_{1}\left\|g_{k+1}\right\|+2\left(1-\eta_{1}\right)\left\|g_{k+1}\right\|\left\|y_{k}^{*}\right\|\left\|d_{k}\right\| / \delta \\
& \leq \eta_{1}\left\|g_{k+1}\right\|+2\left(1-\eta_{1}\right)\left\|g_{k+1}\right\|\left\|y_{k}^{*}\right\|\left\|d_{k}\right\| /\left(\eta_{2}\left\|y_{k}^{*}\right\|\left\|d_{k}\right\|\right) \\
& =\left(\eta_{1}+2\left(1-\eta_{1}\right) / \eta_{2}\right)\left\|g_{k+1}\right\| .
\end{aligned}
$$

Thus, the statement is proved.

Similarly to (3.1) and (3.2), the algorithm has a sufficient descent feature and a trust region trait. To obtain the global convergence, we propose the following necessary assumptions.

\section{Assumption 1}

(i) The level set of $\pi=\left\{x \mid f(x) \leq f\left(x_{0}\right)\right\}$ is bounded.

(ii) The objective function $f \in C^{2}$ is bounded from below, and its gradient function $g$ is Lipschitz continuous, thats is, there exists a constant $\zeta$ such that

$$
\|g(x)-g(y)\| \leq \zeta\|x-y\|, \quad x, y \in R^{n}
$$

The existence and necessity of the step length $\alpha_{k}$ are established in [43]. In view of the discussion and established technique, the global convergence of the proposed algorithm is expressed as follows.

Theorem 3.1 If Assumptions (i)-(ii) are satisfied and the relative sequences of $\left\{x_{k}\right\},\left\{d_{k}\right\}$, $\left\{g_{k}\right\}$, and $\left\{\alpha_{k}\right\}$ are generated by Algorithm 2.1, then

$$
\lim _{k \rightarrow \infty}\left\|g_{k}\right\|=0
$$

Proof By (1.7), (3.1), and (3.3) we have

$$
\begin{aligned}
f\left(x_{k}+\alpha_{k} d_{k}\right) & \leq f_{k}+\iota \alpha_{k} g_{k}^{T} d_{k}+\alpha_{k} \min \left[-\iota_{1} g_{k}^{T} d_{k}, \iota \alpha_{k}\left\|d_{k}\right\|^{2} / 2\right] \\
& \leq f_{k}+\iota \alpha_{k} g_{k}^{T} d_{k}-\alpha_{k} \iota_{1} g_{k}^{T} d_{k} \\
& \leq f_{k}+\alpha_{k}\left(\iota-\iota_{1}\right) g_{k}^{T} d_{k} \\
& \leq f_{k}-\eta_{1} \alpha_{k}\left(\iota-\iota_{1}\right)\left\|g_{k}\right\|^{2} .
\end{aligned}
$$

Summing these inequalities from $k=0$ to $\infty$, under Assumption (ii), we obtain

$$
\sum_{k=0}^{\infty} \eta_{1} \alpha_{k}\left(\iota-\iota_{1}\right)\left\|g_{k}\right\|^{2} \leq f\left(x_{0}\right)-f_{\infty}<+\infty
$$

This means that

$$
\lim _{k \rightarrow \infty} \alpha_{k}\left\|g_{k}\right\|^{2}=0
$$


Similarly to (1.8) and (3.1), we obtain

$$
\begin{aligned}
g\left(x_{k}+\alpha_{k} d_{k}\right)^{T} d_{k} & \geq \tau g_{k}^{T} d_{k}+\min \left[-\iota_{1} g_{k}^{T} d_{k}, \iota \alpha_{k}\left\|d_{k}\right\|^{2}\right] \\
& \geq \tau g_{k}^{T} d_{k} .
\end{aligned}
$$

Thus, we obtain the following inequality:

$$
\begin{aligned}
-\eta_{1}(\tau-1)\left\|g_{k}\right\|^{2} & \leq(\tau-1) g_{k}^{T} d_{k} \\
& \leq\left[g\left(x_{k}+\alpha_{k} d_{k}\right)-g\left(x_{k}\right)\right]^{T} d_{k} \\
& \leq\left\|g\left(x_{k}+\alpha_{k} d_{k}\right)-g\left(x_{k}\right)\right\|\left\|d_{k}\right\| \\
& \leq \alpha_{k} \zeta\left\|d_{k}\right\|^{2},
\end{aligned}
$$

where the last inequality is obtained since the gradient function is Lipschitz continuous. Then, we have

$$
\alpha_{k} \geq \frac{(1-\tau) \eta_{1}\left\|g_{k}\right\|^{2}}{\zeta\left\|d_{k}\right\|^{2}} \geq \frac{(1-\tau) \eta_{1}\left\|g_{k}\right\|^{2}}{\left.\left(\zeta\left(\eta_{1}+2\left(1-\eta_{1}\right) / \eta_{2}\right)^{2}\left\|g_{k}\right\|^{2}\right)\right)}=\frac{(1-\tau) \eta_{1}}{\left(\zeta\left(\eta_{1}+2\left(1-\eta_{1}\right) / \eta_{2}\right)^{2}\right)} .
$$

By (3.6) we arrive at the conclusion

$$
\lim _{k \rightarrow \infty}\left\|g_{k}\right\|^{2}=0
$$

as claimed.

\section{Numerical results}

In this section, we list the numerical result in terms of the algorithm characteristics NI, NFG, and CPU, where NI is the total iteration number, NFG is the sum of the calculation frequency of the objective function and gradient function, and CPU is the calculation time in seconds.

\subsection{Problems and test experiments}

The tested problems listed in Table 1 stem from [48]. At the same time, we introduce two different algorithms into this section to measure the objective algorithm efficiency through the tested problems. We denote the two algorithms as Algorithm 2 and Algorithm 3. They are different from Algorithm 2.1 only at Step 5. One is determined by (1.10), and the other is computed by (1.11).

Stopping rule: If the inequality $\left|f\left(x_{k}\right)\right|>e_{1}$ is correct, let stop $1=\frac{\left|f\left(x_{k}\right)-f\left(x_{k+1}\right)\right|}{\left|f\left(x_{k}\right)\right|}$ or stop $1=$ $\left|f\left(x_{k}\right)-f\left(x_{k+1}\right)\right|$. The algorithm stops when one of the following conditions is satisfied: $\|g(x)\|<\epsilon$, the iteration number is greater than 2000, or stop $1<e_{2}$, where $e_{1}=e_{2}=10^{-5}$ and $\epsilon=10^{-6}$. In Table 1 , "No" and "problem" represent the index of the the tested problems and the name of the problem, respectively.

Initiation: $\iota=0.3, \iota_{1}=0.1, \tau=0.65, \eta_{1}=0.65, \eta_{2}=0.001, \eta_{3}=0.001, \eta_{4}=0.001, \eta_{5}=0.1$.

Dimension: 1200, 3000, 6000, 9000.

Calculation environment: The calculation environment is a computer with $2 \mathrm{~GB}$ of memory, a Pentium(R) Dual-Core CPU E5800@3.20 GHz, and the 64-bit Windows 7 operation system. 
Table 1 Test problems

\begin{tabular}{|c|c|}
\hline No. & Problem \\
\hline 1 & Extended Freudenstein and Roth Function \\
\hline 2 & Extended Trigonometric Function \\
\hline 3 & Extended Rosenbrock Function \\
\hline 4 & Extended White and Holst Function \\
\hline 5 & Extended Beale Function \\
\hline 6 & Extended Penalty Function \\
\hline 7 & Perturbed Quadratic Function \\
\hline 8 & Raydan 1 Function \\
\hline 9 & Raydan 2 Function \\
\hline 10 & Diagonal 1 Function \\
\hline 11 & Diagonal 2 Function \\
\hline 12 & Diagonal 3 Function \\
\hline 13 & Hager Function \\
\hline 14 & Generalized Tridiagonal 1 Function \\
\hline 15 & Extended Tridiagonal 1 Function \\
\hline 16 & Extended Three Exponential Terms Function \\
\hline 17 & Generalized Tridiagonal 2 Function \\
\hline 18 & Diagonal 4 Function \\
\hline 19 & Diagonal 5 Function \\
\hline 20 & Extended Himmelblau Function \\
\hline 21 & Generalized PSC1 Function \\
\hline 22 & Extended PSC1 Function \\
\hline 23 & Extended Powell Function \\
\hline 24 & Extended Block Diagonal BD1 Function \\
\hline 25 & Extended Maratos Function \\
\hline 26 & Extended Cliff Function \\
\hline 27 & Quadratic Diagonal Perturbed Function \\
\hline 28 & Extended Wood Function \\
\hline 29 & Extended Hiebert Function \\
\hline 30 & Quadratic Function QF1 Function \\
\hline 31 & Extended Quadratic Penalty QP1 Function \\
\hline 32 & Extended Quadratic Penalty QP2 Function \\
\hline 33 & A Quadratic Function QF2 Function \\
\hline 34 & Extended EP1 Function \\
\hline 35 & Extended Tridiagonal-2 Function \\
\hline 36 & BDQRTIC Function (CUTE) \\
\hline 37 & TRIDIA Function (CUTE) \\
\hline
\end{tabular}

\begin{tabular}{ll}
\hline No. & Problem \\
\hline 38 & ARWHEAD Function (CUTE) \\
38 & ARWHEAD Function (CUTE) \\
40 & NONDQUAR Function (CUTE) \\
41 & DQDRTIC Function (CUTE) \\
42 & EG2 Function (CUTE) \\
43 & DIXMAANA Function (CUTE) \\
44 & DIXMAANB Function (CUTE) \\
45 & DIXMAANC Function (CUTE) \\
46 & DIXMAANE Function (CUTE) \\
47 & Partial Perturbed Quadratic Function \\
48 & Broyden Tridiagonal Function \\
49 & Almost Perturbed Quadratic Function \\
50 & Tridiagonal Perturbed Quadratic Function \\
51 & EDENSCH Function (CUTE) \\
52 & VARDIM Function (CUTE) \\
53 & STAIRCASE S1 Function \\
54 & LIARWHD Function (CUTE) \\
55 & DIAGONAL 6 Function \\
56 & DIXON3DQ Function (CUTE) \\
57 & DIXMAANF Function (CUTE) \\
58 & DIXMAANG Function (CUTE) \\
59 & DIXMAANH Function (CUTE) \\
60 & DIXMAANI Function (CUTE) \\
61 & DIXMAANJ Function (CUTE) \\
62 & DIXMAANK Function (CUTE) \\
63 & DIXMAANL Function (CUTE) \\
64 & DIXMAAND Function (CUTE) \\
65 & ENGVAL1 Function (CUTE) \\
66 & FLETCHCR Function (CUTE) \\
67 & COSINE Function (CUTE) \\
68 & EXtended DENSCHNB Function (CUTE) \\
69 & DENSCHNF Function (CUTE) \\
70 & SINQUAD Function (CUTE) \\
71 & BIGGSB1 Function (CUTE) \\
72 & Partial Perturbed Quadratic PPQ2 Function \\
73 & Scaled Quadratic SQ1 Function \\
& \\
\hline & \\
\hline
\end{tabular}

A list of the numerical results with the corresponding problem index is listed in Table 2. Then, based on the technique in [49], the plots of the corresponding figures are presented for the three discussed algorithms.

Other case: To save the paper space, we only list the data of dimension of 9000, and the remaining data are listed in the attachment.

\subsection{Results and discussion}

Obviously, the objective algorithm (Algorithm 2.1) is more effective than the other algorithms since the point value on the algorithm curve is largest among the three curves. In Fig. 1, the proposed algorithm curve is above the other curves. This means that the objective algorithm solves complex problems with fewer iterations, and Algorithm 3 is better than Algorithm 2. In Fig. 2, we obtain that the proposed algorithm has a large initial point, which means that it has high efficiency and its curve seems smoother than others. It is well known that the most important metric of an algorithm is the calculation time (CPU time), which is an essential aspect to measure the efficiency of an algorithm. Based on Fig. 3, the 
Table 2 Numerical results

\begin{tabular}{|c|c|c|c|c|c|c|c|c|c|c|}
\hline \multirow[t]{2}{*}{ NO } & \multirow[t]{2}{*}{ Dim } & \multicolumn{3}{|c|}{ Algorithm 2.1} & \multicolumn{3}{|c|}{ Algorithm 2} & \multicolumn{3}{|c|}{ Algorithm 3} \\
\hline & & $\mathrm{NI}$ & NFG & $\mathrm{CPU}$ & $\mathrm{NI}$ & NFG & $\mathrm{CPU}$ & $\mathrm{NI}$ & NFG & $\mathrm{CPU}$ \\
\hline 1 & 9000 & 4 & 20 & 0.124801 & 14 & 48 & 0.405603 & 5 & 26 & 0.249602 \\
\hline 2 & 9000 & 71 & 327 & 1.965613 & 27 & 89 & 0.670804 & 32 & 136 & 0.858005 \\
\hline 3 & 9000 & 7 & 20 & 0.0312 & 37 & 160 & 0.249602 & 27 & 147 & 0.202801 \\
\hline 4 & 9000 & 12 & 49 & 0.280802 & 34 & 161 & 0.717605 & 42 & 219 & 0.951606 \\
\hline 5 & 9000 & 13 & 56 & 0.202801 & 20 & 63 & 0.249602 & 5 & 24 & 0.0624 \\
\hline 6 & 9000 & 65 & 252 & 0.421203 & 43 & 143 & 0.280802 & 3 & 9 & 0.0312 \\
\hline 7 & 9000 & 11 & 37 & 0.0624 & 478 & 979 & 2.215214 & 465 & 1479 & 2.558416 \\
\hline 8 & 9000 & 5 & 20 & 0.0624 & 22 & 55 & 0.156001 & 14 & 54 & 0.156001 \\
\hline 9 & 9000 & 6 & 16 & 0.0312 & 5 & 21 & 0.0624 & 3 & 8 & 0.0312 \\
\hline 10 & 9000 & 2 & 13 & 0.0156 & 2 & 13 & 0.000001 & 2 & 13 & 0.000001 \\
\hline 11 & 9000 & 3 & 17 & 0.0312 & 7 & 34 & 0.0624 & 17 & 87 & 0.218401 \\
\hline 12 & 9000 & 3 & 10 & 0.0312 & 19 & 40 & 0.202801 & 14 & 50 & 0.202801 \\
\hline 13 & 9000 & 3 & 24 & 0.0624 & 3 & 24 & 0.0312 & 3 & 24 & 0.0156 \\
\hline 14 & 9000 & 4 & 12 & 4.305628 & 5 & 14 & 5.382034 & 5 & 14 & 5.226033 \\
\hline 15 & 9000 & 19 & 77 & 9.984064 & 22 & 66 & 9.516061 & 21 & 71 & 10.296066 \\
\hline 16 & 9000 & 3 & 11 & 0.0624 & 6 & 27 & 0.078 & 6 & 18 & 0.0624 \\
\hline 17 & 9000 & 11 & 45 & 0.374402 & 27 & 69 & 0.780005 & 27 & 87 & 0.811205 \\
\hline 18 & 9000 & 5 & 23 & 0.0312 & 3 & 10 & 0.000001 & 3 & 10 & 0.0312 \\
\hline 19 & 9000 & 3 & 9 & 0.0624 & 3 & 9 & 0.0312 & 3 & 19 & 0.0312 \\
\hline 20 & 9000 & 19 & 76 & 0.124801 & 15 & 36 & 0.0624 & 3 & 9 & 0.0312 \\
\hline 21 & 9000 & 12 & 47 & 0.156001 & 13 & 61 & 0.187201 & 15 & 59 & 0.218401 \\
\hline 22 & 9000 & 7 & 46 & 0.795605 & 8 & 70 & 0.577204 & 6 & 46 & 0.686404 \\
\hline 23 & 9000 & 9 & 45 & 0.218401 & 101 & 357 & 2.090413 & 46 & 150 & 0.873606 \\
\hline 24 & 9000 & 5 & 47 & 0.093601 & 14 & 88 & 0.156001 & 14 & 97 & 0.249602 \\
\hline 25 & 9000 & 9 & 28 & 0.0312 & 40 & 214 & 0.249602 & 8 & 46 & 0.0624 \\
\hline 26 & 9000 & 24 & 102 & 0.327602 & 24 & 100 & 0.249602 & 3 & 24 & 0.0312 \\
\hline 27 & 9000 & 6 & 20 & 0.0312 & 34 & 109 & 0.187201 & 92 & 321 & 0.530403 \\
\hline 28 & 9000 & 13 & 50 & 0.124801 & 20 & 83 & 0.109201 & 23 & 84 & 0.140401 \\
\hline 29 & 9000 & 6 & 36 & 0.0468 & 4 & 21 & 0.0312 & 4 & 21 & 0.0312 \\
\hline 30 & 9000 & 11 & 37 & 0.0624 & 454 & 931 & 1.450809 & 424 & 1346 & 1.747211 \\
\hline 31 & 9000 & 18 & 63 & 0.124801 & 15 & 51 & 0.093601 & 3 & 10 & 0.0312 \\
\hline 32 & 9000 & 18 & 70 & 0.218401 & 23 & 61 & 0.218401 & 3 & 18 & 0.0624 \\
\hline 33 & 9000 & 2 & 5 & 0.000001 & 2 & 5 & 0.0312 & 2 & 5 & 0.000001 \\
\hline 34 & 9000 & 8 & 16 & 0.0312 & 6 & 12 & 0.0312 & 3 & 6 & 0.0312 \\
\hline 35 & 9000 & 4 & 13 & 0.0312 & 4 & 10 & 0.0312 & 3 & 8 & 0.000001 \\
\hline 36 & 9000 & 7 & 23 & 4.602029 & 8 & 28 & 5.569236 & 10 & 47 & 8.673656 \\
\hline 37 & 9000 & 7 & 23 & 0.0624 & 1412 & 2829 & 6.942044 & 2000 & 6021 & 11.356873 \\
\hline 38 & 9000 & 4 & 18 & 0.0312 & 8 & 35 & 0.187201 & 4 & 11 & 0.0312 \\
\hline 39 & 9000 & 5 & 19 & 0.0312 & 28 & 56 & 0.124801 & 3 & 8 & 0.0312 \\
\hline 40 & 9000 & 13 & 43 & 0.561604 & 835 & 2936 & 36.223432 & 9 & 41 & 0.421203 \\
\hline 41 & 9000 & 10 & 32 & 0.0624 & 17 & 41 & 0.093601 & 22 & 81 & 0.124801 \\
\hline 42 & 9000 & 4 & 33 & 0.0624 & 13 & 35 & 0.124801 & 9 & 47 & 0.109201 \\
\hline 43 & 9000 & 16 & 62 & 1.029607 & 16 & 38 & 0.951606 & 13 & 48 & 0.780005 \\
\hline 44 & 9000 & 3 & 17 & 0.156001 & 9 & 50 & 0.624004 & 3 & 17 & 0.187201 \\
\hline 45 & 9000 & 21 & 118 & 1.49761 & 12 & 81 & 0.858006 & 3 & 24 & 0.202801 \\
\hline 46 & 9000 & 20 & 81 & 1.435209 & 209 & 443 & 11.247672 & 110 & 362 & 6.630042 \\
\hline 47 & 9000 & 11 & 37 & 27.066173 & 30 & 97 & 68.64044 & 37 & 112 & 87.220159 \\
\hline 48 & 9000 & 13 & 54 & 9.718862 & 31 & 92 & 18.610919 & 23 & 50 & 11.980877 \\
\hline 49 & 9000 & 11 & 37 & 0.0624 & 478 & 979 & 1.51321 & 504 & 1592 & 1.887612 \\
\hline 50 & 9000 & 11 & 37 & 7.971651 & 472 & 967 & 263.68849 & 444 & 1273 & 299.381519 \\
\hline 51 & 9000 & 6 & 31 & 0.156001 & 7 & 25 & 0.218401 & 3 & 17 & 0.124801 \\
\hline 52 & 9000 & 62 & 186 & 0.998406 & 63 & 195 & 0.842405 & 4 & 21 & 0.0624 \\
\hline 53 & 9000 & 10 & 32 & 0.0312 & 2000 & 4059 & 7.72205 & 1865 & 5618 & 7.971651 \\
\hline 54 & 9000 & 4 & 11 & 0.0312 & 21 & 79 & 0.156001 & 17 & 79 & 0.124801 \\
\hline 55 & 9000 & 10 & 24 & 3.010819 & 7 & 25 & 3.213621 & 3 & 10 & 1.076407 \\
\hline 56 & 9000 & 7 & 21 & 0.0156 & 2000 & 4003 & 6.489642 & 1390 & 4107 & 5.335234 \\
\hline 57 & 9000 & 5 & 39 & 0.358802 & 67 & 220 & 4.024826 & 3 & 24 & 0.202801 \\
\hline 58 & 9000 & 5 & 24 & 0.343202 & 114 & 282 & 6.411641 & 82 & 315 & 5.257234 \\
\hline 59 & 9000 & 5 & 39 & 0.343202 & 68 & 310 & 4.72683 & 3 & 23 & 0.171601 \\
\hline
\end{tabular}


Table 2 (Continued)

\begin{tabular}{|c|c|c|c|c|c|c|c|c|c|c|}
\hline \multirow[t]{2}{*}{ NO } & \multirow[t]{2}{*}{ Dim } & \multicolumn{3}{|c|}{ Algorithm 2.1} & \multicolumn{3}{|c|}{ Algorithm 2} & \multicolumn{3}{|c|}{ Algorithm 3} \\
\hline & & $\mathrm{NI}$ & NFG & CPU & $\mathrm{NI}$ & NFG & CPU & $\mathrm{NI}$ & NFG & CPU \\
\hline 60 & 9000 & 18 & 74 & 1.294808 & 206 & 437 & 11.107271 & 119 & 363 & 6.957645 \\
\hline 61 & 9000 & 5 & 39 & 0.358802 & 85 & 247 & 4.929632 & 3 & 24 & 0.218401 \\
\hline 62 & 9000 & 4 & 32 & 0.234001 & 4 & 32 & 0.249602 & 3 & 22 & 0.187201 \\
\hline 63 & 9000 & 3 & 22 & 0.187201 & 3 & 22 & 0.187201 & 3 & 22 & 0.187201 \\
\hline 64 & 9000 & 5 & 39 & 0.343202 & 23 & 147 & 1.747211 & 3 & 23 & 0.218401 \\
\hline 65 & 9000 & 12 & 59 & 15.334898 & 14 & 51 & 14.944896 & 7 & 21 & 6.130839 \\
\hline 66 & 9000 & 3 & 9 & 1.62241 & 2000 & 4022 & 1114.767546 & 529 & 2196 & 443.526443 \\
\hline 67 & 9000 & 5 & 28 & 0.093601 & 15 & 58 & 0.280802 & 3 & 23 & 0.0312 \\
\hline 68 & 9000 & 13 & 55 & 0.109201 & 11 & 27 & 0.0624 & 9 & 25 & 0.0624 \\
\hline 69 & 9000 & 16 & 73 & 0.218401 & 24 & 55 & 0.187201 & 20 & 70 & 0.171601 \\
\hline 70 & 9000 & 4 & 13 & 2.542816 & 41 & 203 & 36.332633 & 35 & 231 & 37.783442 \\
\hline 71 & 9000 & 11 & 35 & 0.093601 & 2000 & 4014 & 6.708043 & 1491 & 4631 & 5.600436 \\
\hline 72 & 9000 & 9 & 30 & 21.85574 & 1089 & 3897 & 2675.588751 & 287 & 1015 & 704.391315 \\
\hline 73 & 9000 & 19 & 65 & 0.093601 & 607 & 1269 & 1.856412 & 669 & 2062 & 2.293215 \\
\hline
\end{tabular}

Figure 1 Performance profiles of these methods (NI)

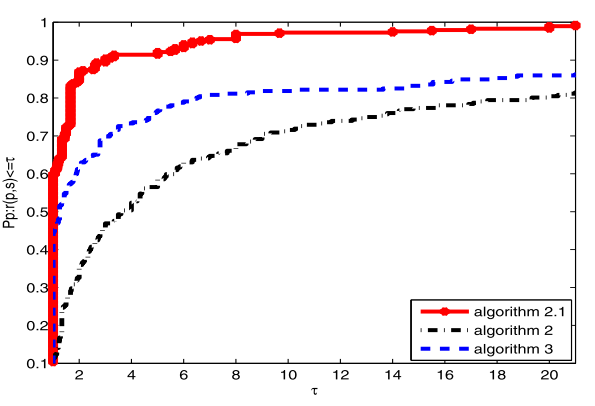

Figure 2 Performance profiles of these methods (NFG)

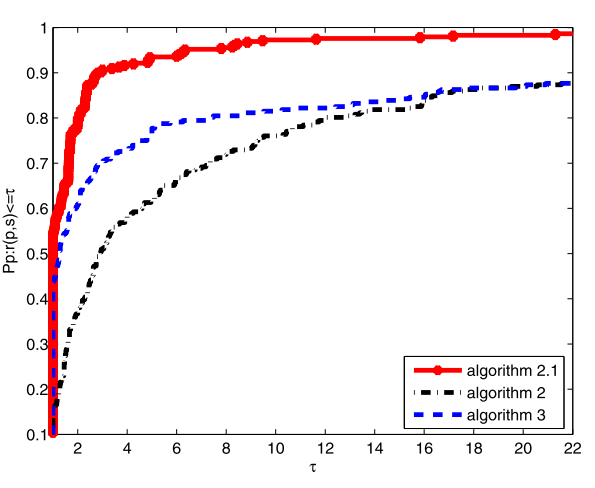

objective algorithm successfully fully utilizes its outstanding characteristics. Therefore, it saves time compared to the other algorithms in addressing complex problems.

\section{Nonlinear equations}

The model of nonlinear equations is given by

$$
h(x)=0,
$$


Figure 3 Performance profiles of these methods (CPU time)

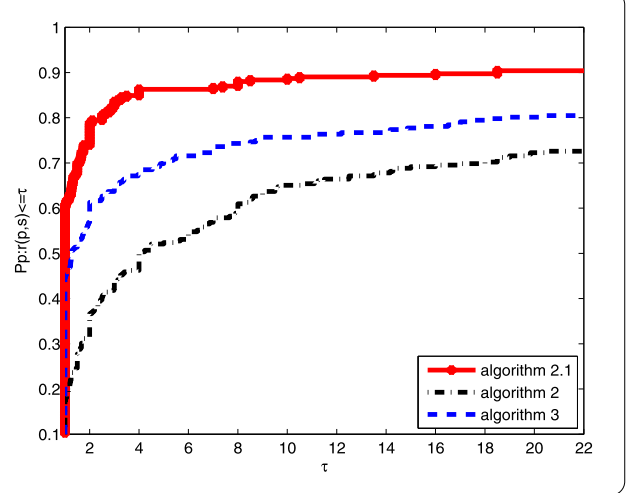

where the function of $h$ is continuously differentiable and monotonous, and $x \in R^{n}$, that is,

$$
(h(x)-h(y))(x-y)>0, \quad \forall x, y \in R^{n} .
$$

Scholars and writers paid much attention to this model since it significantly influences various fields such as physics and computer technology (see [1-3, 8-11]), and it has resulted in many fruitful theories and good techniques (see [47, 50-54]). By mathematical calculations we obtain that (5.1) is equivalent to the model

$$
\min F(x)
$$

where $F(x)=\frac{\|h(x)\|^{2}}{2}$, and $\|\cdot\|$ is the Euclidean norm. Then, we pay much attention to the mathematical model (5.2) since (5.1) and (5.2) have the same solution. In general, the mathematical formula for (5.2) is $x_{k+1}=x_{k}+\alpha_{k} d_{k}$. Now, we introduce the following famous line search technique into this paper $[47,55]$ :

$$
-h\left(x_{k}+\alpha_{k} d_{k}\right)^{T} d_{k} \geq \sigma \alpha_{k}\left\|h\left(x_{k}+\alpha_{k} d_{k}\right)\right\|\left\|d_{k}\right\|^{2}
$$

where $\alpha_{k}=\max \left\{s, s \rho, s \rho^{2}, \ldots\right\}, s, \rho>0, \rho \in(0,1)$, and $\sigma>0$. Solodov [56] proposes a projection proximal point algorithm in a Hilbert space that finds the zeros of set-valued maximal monotone operators. Ceng and Yao [57-60] paid much attention to the research in Hilbert spaces and obtained successful achievements. Solodov and Svaiter [61] applied the projection technique to large-scale nonlinear equations and obtained some ideal achievements. For the projection-based technique, the famous formula

$$
h\left(w_{k}\right)^{T}\left(x_{k}-w_{k}\right)>0
$$

is flexible, where $w_{k}=x_{k}+\alpha_{k} d_{k}$. The search direction is extremely important for the proposed algorithm since it largely determines the efficiency. Likewise, the algorithm contains the perfect line search technique. By the monotonicity of $h(x)$ we obtain

$$
h\left(w_{k}\right)^{T}\left(x^{*}-w_{k}\right) \leq 0
$$


$\overline{\text { Algorithm 5.1 Modified three-term conjugate gradient algorithm for large-scale nonlin- }}$ ear equations

Step 1: Choose an initial point $x_{1} \in R^{n}, \sigma>0, s>0, \rho \in(0,1), \eta_{i}>0(i=1,2,3,4,5)$, and positive constants $\varepsilon \in(0,1)$. Let $k=1$.

Step 2: Stop if $\left\|h\left(x_{k}\right)\right\| \leq \varepsilon$. Otherwise, calculate $d_{k+1}$ similar to (5.6).

Step 3: Find the step length $\alpha_{k}$ similar to (5.3).

Step 4: Reset the new iteration point of $w_{k}=x_{k}+\alpha_{k} d_{k}$.

Step 5: If $\left\|h\left(w_{k}\right)\right\| \leq \varepsilon$, then stop and set $x_{k+1}=w_{k}$. Otherwise, calculate $x_{k+1}$ similar to (5.5).

Step 6: Let $k:=k+1$ and return to Step 2 .

where $x^{*}$ is the solution of $h\left(x^{*}\right)=0$. We consider the hyperplane

$$
\Lambda=\left\{x \in R^{n} \mid h\left(w_{k}\right)^{T}\left(x-w_{k}\right)=0\right\} .
$$

It is obvious that the hyperplane separates the current iteration point of $x_{k}$ from the zeros of the mathematical model (5.1). Then, we need to calculate the next iteration point $x_{k+1}$ through projection of current point $x_{k}$. Therefore, we give the following formula for the next point:

$$
x_{k+1}=x_{k}-\frac{h\left(w_{k}\right)^{T}\left(x_{k}-w_{k}\right) h\left(w_{k}\right)}{\left\|h\left(w_{k}\right)^{2}\right\|} .
$$

In [55], it is proved that formula (5.5) is effective since it not only obtains perfect numerical results but also has perfect theoretical characteristics. Thus, we introduce it here. The formula of the search direction $d_{k+1}$ is given by

$$
d_{k+1}= \begin{cases}-\eta_{1} h_{k+1}+\left(1-\eta_{1}\right)\left(d_{k}^{T} h_{k+1} y_{k}^{*}-h_{k+1}^{T} y_{k}^{*} d_{k}\right) / \delta & \text { if } k \geq 1, \\ -h_{k+1} & \text { if } k=0,\end{cases}
$$

where $\delta=\max \left(\min \left(\eta_{5}\left|s_{k}^{T} y_{k}^{*}\right|,\left|d_{k}^{T} y_{k}^{*}\right|\right), \eta_{2}\left\|y_{k}^{*}\right\|\left\|d_{k}\right\|, \eta_{3}\left\|g_{k}\right\|^{2}\right)+\eta_{4} *\left\|d_{k}\right\|^{2}, y_{k}^{*}=h_{k+1}-\frac{\left\|h_{k+1}\right\|^{2}}{\left\|h_{k}\right\|^{2}} h_{k}$, and $\eta_{i}>0(i=1,2,3)$. Now, we express the specific content of the proposed algorithm.

\section{The global convergence of Algorithm 5.1}

First, we make the following necessary assumptions.

\section{Assumption 2}

(i) The objective model of (5.1) has a nonempty solution set.

(ii) The function $h$ is Lipschitz continuous on $R^{n}$, which means that there is a positive constant $L$ such that

$$
\|h(x)-h(y)\| \leq L\|x-y\|, \quad \forall x, y \in R^{n} .
$$

By Assumption 2(ii) it is obvious that

$$
\left\|h_{k}\right\| \leq \theta
$$


where $\theta$ is a positive constant. Then, the necessary properties of the search direction are the following (we omit the proof):

$$
h_{k}^{T} d_{k}=-\eta_{1}\left\|h_{k}\right\|\left\|h_{k}\right\|
$$

and

$$
\left\|d_{k}\right\| \leq\left(\eta_{1}+2\left(1-\eta_{1}\right) / \eta_{2}\right)\left\|h_{k}\right\| .
$$

Now, we give some lemmas, which we utilize to obtain the global convergence of the proposed algorithm.

Lemma 6.1 If Assumption 2 holds, the relevant sequence $\left\{x_{k}\right\}$ is produced by Algorithm 5.1, and the point $x^{*}$ is the solution of the objective model (5.1). We obtain that the formula

$$
\left\|x_{k+1}-x^{*}\right\|^{2} \leq\left\|x_{k}-x^{*}\right\|^{2}-\left\|x_{k+1}-x_{k}\right\|^{2}
$$

is correct and the sequence $\left\{x_{k}\right\}$ is bounded. Furthermore, either the last iteration point is the solution of the objective model and the sequence of $\left\{x_{k}\right\}$ is bounded, or the sequence of $\left\{x_{k}\right\}$ is infinite and satisfies the condition

$$
\sum_{k=0}^{\infty}\left\|x_{k+1}-x_{k}\right\|^{2}<\infty
$$

This paper merely proposes, but omits, the relevant proof since it is similar to the proof in [61].

Lemma 6.2 Algorithm 5.1 generates an iteration point in a finite number of iteration steps, which satisfies the formula of $x_{k+1}=x_{k}+\alpha_{k} d_{k}$ if Assumption 2 holds.

Proof We denote $\Psi=N \cup\{0\}$. We suppose that Algorithm 5.1 has terminated or the formula $\left\|h_{k}\right\| \rightarrow 0$ is erroneous. This means that there exists a constant $\varepsilon_{*}$ such that

$$
\left\|h_{k}\right\| \geq \varepsilon_{*}, \quad k \in \Psi
$$

We prove this conclusion by contradiction. Suppose that certain iteration indexes $k^{*}$ fail to meet the condition (5.3) of the line search technique. Without loss of generality, we denote the corresponding step length as $\alpha_{k^{*}}^{(l)}$, where $\alpha_{k^{*}}^{(l)}=\rho^{l} s$. This means that

$$
-h\left(x_{k^{*}}+\alpha_{k^{*}}^{(l)} d_{k^{*}}\right)^{T} d_{k^{*}}<\sigma \alpha_{k^{*}}^{(l)}\left\|h\left(x_{k^{*}}+\alpha_{k^{*}}^{(l)} d_{k^{*}}\right)\right\|\left\|d_{k^{*}}\right\|^{2} .
$$

By (6.3) and Assumption 2(ii) we obtain

$$
\begin{aligned}
\left\|h_{k^{*}}\right\|^{2} & =-\eta_{1} h_{k^{*}}^{T} d_{k^{*}} \\
& =\eta_{1}\left[\left(h\left(x_{k^{*}}+\alpha_{k^{*}}^{(l)} d_{k^{*}}\right)-h\left(x_{k^{*}}\right)\right)^{T} d_{k^{*}}-\left(h\left(x_{k^{*}}+\alpha_{k^{*}}^{(l)} d_{k^{*}}\right)^{T} d_{k^{*}}\right)\right] \\
& <\eta_{1}\left[L+\sigma\left\|h\left(x_{k^{*}}+\alpha_{k^{*}}^{(l)} d_{k^{*}}\right)\right\|\right] \alpha_{k^{*}}^{(l)}\left\|d_{k^{*}}\right\|^{2}, \quad \forall l \in \Psi .
\end{aligned}
$$


By (6.3) and (6.4) we have

$$
\begin{aligned}
\left\|h\left(x_{k^{*}}+\alpha_{k^{*}}^{(l)} d_{k^{*}}\right)\right\| & \leq\left\|h\left(x_{k^{*}}+\alpha_{k^{*}}^{(l)} d_{k^{*}}\right)-h\left(x_{k^{*}}\right)\right\|+\left\|h\left(x_{k^{*}}\right)\right\| \\
& \leq L \alpha_{k^{*}}^{(l)}\left\|d_{k^{*}}\right\|+\theta \\
& \leq \operatorname{Ls} \theta\left(\eta_{1}+2\left(1-\eta_{1}\right) / \eta_{2}\right)+\theta
\end{aligned}
$$

By (6.6) we obtain

$$
\begin{aligned}
\alpha_{k^{*}}^{(l)} & >\frac{\left\|h_{k^{*}}\right\|^{2}}{\eta_{1}\left[L+\sigma\left\|h\left(x_{k^{*}}+\alpha_{k^{*}}^{(l)} d_{k^{*}}\right)\right\|\right]\left\|d_{k^{*}}\right\|^{2}} \\
& >\frac{\left\|h_{k^{*}}\right\|^{2}}{\eta_{1}\left[L+\sigma\left(L s \theta\left(\eta_{1}+2\left(1-\eta_{1}\right) / \eta_{2}\right)+\theta\right)\right]\left\|d_{k^{*}}\right\|^{2}} \\
& >\frac{\eta_{2}^{2}}{\eta_{1}\left[L+\sigma\left(L s \theta\left(\eta_{1}+2 / \eta_{3}\right)+\theta\right)\right]\left(2\left(1-\eta_{1}\right)+\eta_{2} \eta_{1}\right)^{2}}, \quad \forall l \in \Psi .
\end{aligned}
$$

It is obvious that this formula fails to meet the definition of the step length $\alpha_{k^{*}}^{(l)}$. Thus, we conclude that the proposed line search technique is reasonable and necessary. In other words, the line search technique generates a positive constant $\alpha_{k}$ in a finite frequency of backtracking repetitions. By the established conclusion we propose the following theorem on the global convergence of the proposed algorithm.

Theorem 6.1 If Assumption 2 holds and the relevant sequences $\left\{d_{k}, \alpha_{k}, x_{k+1}, h_{k+1}\right\}$ are calculated using Algorithm 5.1, then

$$
\liminf _{k \rightarrow \infty}\left\|h_{k}\right\|=0
$$

Proof We prove this by contradiction. This means that there exist a constant $\varepsilon_{0}>0$ and an index $k_{0}$ such that

$$
\left\|h_{k}\right\| \geq \varepsilon_{0}, \quad \forall k \geq k_{0}
$$

On the one hand, by (6.2) and (6.4) we obtain

$$
\left\|d_{k}\right\| \leq\left(\eta_{1}+2\left(1-\eta_{1}\right) / \eta_{2}\right)\left\|h_{k}\right\| \leq\left(\eta_{1}+2\left(1-\eta_{1}\right) / \eta_{2}\right) \theta, \quad \forall k \in \Psi
$$

On the other hand, from (6.3) we have

$$
\left\|d_{k}\right\| \geq \eta_{1}\left\|h_{k}\right\| \geq \eta_{1} \theta
$$

These inequalities indicate that the sequence of $\left\{d_{k}\right\}$ is bounded. This means that there exist an accumulation point $d^{*}$ and the corresponding infinite set $N_{1}$ such that

$$
\lim _{k \rightarrow \infty} d_{k}=d^{*}, \quad k \in N_{1}
$$


By Lemma 6.1 we obtain that the sequence of $\left\{x_{k}\right\}$ is bounded. Thus, there exist an infinite index set $N_{2} \subset N_{1}$ and an accumulation point $x^{*}$ that meet the formula

$$
\lim _{k \rightarrow \infty} x_{k}=x^{*}, \quad \forall k \in N_{2} .
$$

By Lemmas 6.1 and 6.2 we obtain

$$
\alpha_{k}\left\|d_{k}\right\| \rightarrow 0, \quad k \rightarrow \infty
$$

Since $\left\{d_{k}\right\}$ is bounded, we obtain

$$
\lim _{k \rightarrow \infty} \alpha_{k}=0
$$

By the definition of $\alpha_{k}$ we obtain the following inequality:

$$
-h\left(x_{k}+\alpha_{k}^{*} d_{k}\right)^{T} d_{k} \leq \sigma \alpha_{k}^{*}\left\|h\left(x_{k}+\alpha_{k}^{*} d_{k}\right)\right\|\left\|d_{k}\right\|^{2},
$$

where $\alpha_{k}^{*}=\alpha_{k} / \rho$. Now, we take the limit on both sides of (6.10) and (6.3) and obtain

$$
h\left(x^{*}\right)^{T} d^{*}>0
$$

and

$$
h\left(x^{*}\right)^{T} d^{*} \leq 0
$$

The obtained contradiction completes the proof.

\section{The results of nonlinear equations}

In this section, we list the relevant numerical results of nonlinear equations and present the objective function $h(x)=\left(f_{1}(x), f_{2}(x), \ldots, f_{n}(x)\right)$, where the relevant functions' information is listed in Table 1.

\subsection{Problems and test experiments}

To measure the efficiency of the proposed algorithm, in this section, we compare this method with (1.10) (as Algorithm 6) using three characteristics "NI", "NG", and "CPU" and the remind that Algorithm 6 is identical to Algorithm 5.1. "NI" presents the number of iterations, "NG" is the calculation frequency of the function, and "CPU" is the time of the process in addressing the tested problems. In Table 1, "No" and "problem" express the indices and the names of the test problems.

Stopping rule: If $\left\|g_{k}\right\| \leq \varepsilon$ or the whole iteration number is greater than 2000, the algorithm stops.

Initiation: $\varepsilon=1 e-5, \sigma=0.8, s=1, \rho=0.9, \eta_{1}=0.85, \eta_{2}=\eta_{3}=0.001, \eta_{4}=\eta_{5}=0.1$.

Dimension: 3000, 6000, 9000.

Calculation environment: The calculation environment is a computer with 2 GB of memory, a Pentium(R) Dual-Core CPU E5800@3.20 GHz, and the 64-bit Windows 7 operation system. 
Table 3 Test problems

\begin{aligned} & \hline No. Problem \\ & \hline 1 Exponential function 1 \\ & 2 Exponential function 2 \\ & 3 Trigonometric function \\ & 4 Singular function \\ & 5 Logarithmic function \\ & 6 Broyden tridiagonal function \\ & 7 Trigexp function \\ & 8 Strictly convex function 1 \\ & 9 Strictly convex function 2 \\ & 10 Zero Jacobian function \\ & \hline\end{aligned}

\begin{tabular}{ll}
\hline No. & Problem \\
\hline 11 & Linear function-full rank \\
12 & Penalty function \\
13 & Variable dimensioned function \\
14 & Extended Powel singular function \\
15 & Tridiagonal system \\
16 & Five-diagonal system \\
17 & Extended Freudentein and Roth function \\
18 & Extended Wood problem \\
19 & Discrete boundary value problem \\
\hline
\end{tabular}

Figure 4 Performance profiles of these methods (NI)

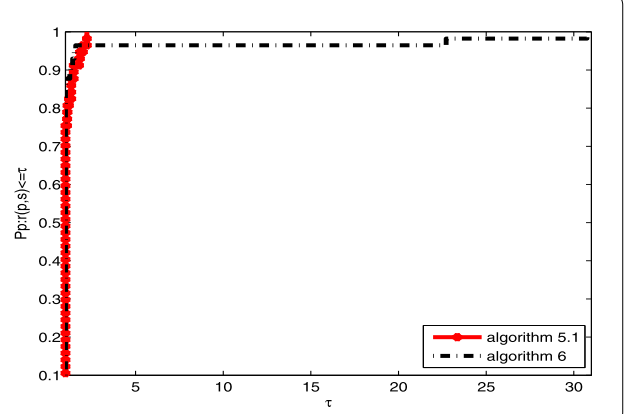

The numerical results with the corresponding problem index are listed in Table 4 . Then, by the technique in [49], the plots of the corresponding figures are presented for two discussed algorithms.

\subsection{Results and discussion}

From the above figures, we safely arrive at the conclusion that the proposed algorithm is perfect compared to similar optimization methods since the algorithm (1.10) is perfect to a large extent. In Fig. 4 we see that the proposed algorithm quickly arrives at a value of 1.0, whereas the left one slowly approaches 1.0. This means that the objective method is successful and efficient for addressing complex problems in our life and work. It is well known that the calculation time is one of the most essential characteristics in an evaluation index of the efficiency of an algorithm. From Figs. 5 and 6, it is obvious that the two algorithms are good since their corresponding point values arrive at 1.0. This result expresses that the above two algorithms solve all of the tested problems and that the proposed algorithm is efficient.

\section{Conclusion}

This paper focuses on the three-term conjugate gradient algorithms and use them to solve the optimization problems and the nonlinear equations. The given method has some good properties.

(i) The proposed three-term conjugate gradient formula possesses the sufficient descent property and the trust region feature without any conditions. The sufficient descent property can make the objective function value be descent, and then the iteration sequence $\left\{x_{k}\right\}$ converges to the global limit point. Moreover, the trust region is good for the proof of the presented algorithm to be easily turned out. 
Table 4 Numerical results

\begin{tabular}{|c|c|c|c|c|c|c|c|}
\hline \multirow[t]{2}{*}{ NO } & \multirow[t]{2}{*}{ Dim } & \multicolumn{3}{|c|}{ Algorithm 5.1} & \multicolumn{3}{|c|}{ Algorithm 6} \\
\hline & & $\overline{\mathrm{NI}}$ & NFG & $\mathrm{CPU}$ & $\mathrm{NI}$ & NFG & $\mathrm{CPU}$ \\
\hline 1 & 3000 & 161 & 162 & 3.931225 & 146 & 147 & 4.149627 \\
\hline 1 & 6000 & 126 & 127 & 12.760882 & 115 & 116 & 11.122871 \\
\hline 1 & 9000 & 111 & 112 & 22.464144 & 99 & 100 & 19.515725 \\
\hline 2 & 3000 & 5 & 76 & 1.185608 & 5 & 76 & 1.060807 \\
\hline 2 & 6000 & 6 & 91 & 4.758031 & 5 & 76 & 4.009226 \\
\hline 2 & 9000 & 5 & 62 & 6.926444 & 5 & 62 & 6.754843 \\
\hline 3 & 3000 & 33 & 228 & 3.276021 & 18 & 106 & 1.778411 \\
\hline 3 & 6000 & 40 & 275 & 15.490899 & 18 & 106 & 6.084039 \\
\hline 3 & 9000 & 40 & 285 & 33.243813 & 18 & 106 & 12.54248 \\
\hline 4 & 3000 & 4 & 61 & 0.842405 & 4 & 61 & 0.936006 \\
\hline 4 & 6000 & 4 & 47 & 2.698817 & 4 & 61 & 3.322821 \\
\hline 4 & 9000 & 4 & 47 & 5.226033 & 4 & 61 & 6.817244 \\
\hline 5 & 3000 & 23 & 237 & 3.244821 & 23 & 237 & 3.354022 \\
\hline 5 & 6000 & 25 & 263 & 14.133691 & 25 & 263 & 13.930889 \\
\hline 5 & 9000 & 26 & 278 & 30.186193 & 26 & 278 & 30.092593 \\
\hline 6 & 3000 & 1999 & 29986 & 382.951255 & 1999 & 29986 & 365.369942 \\
\hline 6 & 6000 & 88 & 1307 & 68.141237 & 1999 & 29986 & 1484.240314 \\
\hline 6 & 9000 & 65 & 962 & 101.806253 & 1999 & 29986 & 3113.998361 \\
\hline 7 & 3000 & 4 & 47 & 0.748805 & 3 & 46 & 0.624004 \\
\hline 7 & 6000 & 4 & 47 & 2.589617 & 3 & 46 & 2.386815 \\
\hline 7 & 9000 & 4 & 47 & 5.257234 & 3 & 46 & 5.054432 \\
\hline 8 & 3000 & 25 & 156 & 2.854818 & 17 & 142 & 1.872012 \\
\hline 8 & 6000 & 32 & 189 & 10.826469 & 18 & 162 & 8.377254 \\
\hline 8 & 9000 & 28 & 192 & 21.512538 & 19 & 174 & 18.938521 \\
\hline 9 & 3000 & 10 & 151 & 1.934412 & 5 & 76 & 1.014007 \\
\hline 9 & 6000 & 4 & 61 & 3.510023 & 5 & 76 & 3.884425 \\
\hline 9 & 9000 & 4 & 61 & 6.614442 & 6 & 91 & 9.609662 \\
\hline 10 & 3000 & 1999 & 29986 & 386.804479 & 1999 & 29986 & 359.816306 \\
\hline 10 & 6000 & 1999 & 29986 & 1523.068963 & 1999 & 29986 & 1469.59182 \\
\hline 10 & 9000 & 1999 & 29986 & 3164.339884 & 1999 & 29986 & 3087.712193 \\
\hline 11 & 3000 & 498 & 7457 & 98.32743 & 499 & 7472 & 93.101397 \\
\hline 11 & 6000 & 498 & 7457 & 385.026068 & 499 & 7472 & 367.787958 \\
\hline 11 & 9000 & 498 & 7457 & 794.07629 & 498 & 7457 & 774.825767 \\
\hline 12 & 3000 & 1999 & 2000 & 51.059127 & 1999 & 2000 & 46.238696 \\
\hline 12 & 6000 & 1999 & 2000 & 199.322478 & 1999 & 2000 & 185.71919 \\
\hline 12 & 9000 & 1999 & 2000 & 405.680601 & 1999 & 2000 & 391.234908 \\
\hline 13 & 3000 & 1 & 2 & 0.0312 & 1 & 2 & 0.0624 \\
\hline 13 & 6000 & 1 & 2 & 0.156001 & 1 & 2 & 0.187201 \\
\hline 13 & 9000 & 1 & 2 & 0.140401 & 1 & 2 & 0.249602 \\
\hline 14 & 3000 & 1999 & 29972 & 400.220565 & 1999 & 29973 & 362.671125 \\
\hline 14 & 6000 & 1999 & 29972 & 1544.316299 & 1999 & 29973 & 1460.294161 \\
\hline 14 & 9000 & 1999 & 29972 & 3197.287295 & 1999 & 29973 & 3105.168705 \\
\hline 15 & 3000 & 4 & 61 & 0.733205 & 4 & 61 & 0.733205 \\
\hline 15 & 6000 & 4 & 61 & 3.790824 & 4 & 61 & 3.026419 \\
\hline 15 & 9000 & 4 & 61 & 6.552042 & 4 & 61 & 6.146439 \\
\hline 16 & 3000 & 5 & 62 & 1.060807 & 5 & 62 & 0.858006 \\
\hline 16 & 6000 & 5 & 62 & 3.400822 & 5 & 62 & 3.291621 \\
\hline 16 & 9000 & 5 & 62 & 6.942044 & 5 & 62 & 6.25564 \\
\hline 17 & 3000 & 6 & 77 & 1.326009 & 6 & 91 & 1.216808 \\
\hline 17 & 6000 & 6 & 77 & 4.243227 & 6 & 91 & 4.570829 \\
\hline 17 & 9000 & 6 & 77 & 8.548855 & 6 & 91 & 9.40686 \\
\hline 18 & 3000 & 5 & 76 & 0.936006 & 5 & 76 & 0.920406 \\
\hline 18 & 6000 & 5 & 76 & 3.900025 & 5 & 76 & 3.775224 \\
\hline 18 & 9000 & 5 & 76 & 8.533255 & 5 & 76 & 7.86245 \\
\hline 19 & 3000 & 108 & 1060 & 15.5689 & 141 & 1272 & 17.565713 \\
\hline 19 & 6000 & 81 & 788 & 44.429085 & 114 & 1029 & 53.820345 \\
\hline 19 & 9000 & 63 & 628 & 70.512452 & 100 & 903 & 99.715839 \\
\hline
\end{tabular}


Figure 5 Performance profiles of these methods (NG)

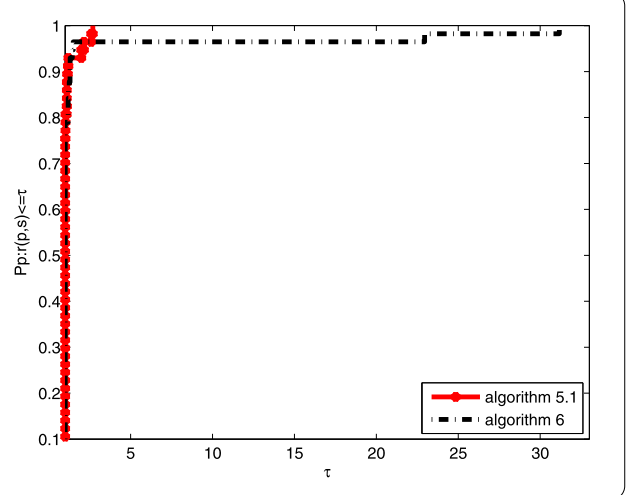

Figure 6 Performance profiles of these methods (CPU time)

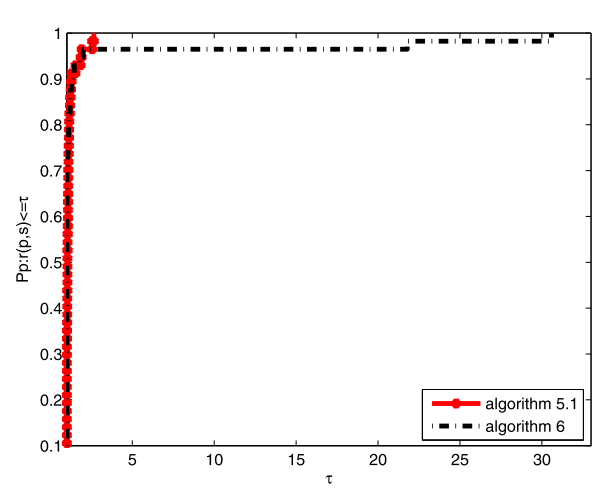

(ii) The given algorithm can be used for not only the normal unstrained optimization problems but also for the nonlinear equations. Both algorithms for these two problems have the global convergence under general conditions.

(iii) Large-scale problems are done by the given problems, which shows that the new algorithms are very effective.

\section{Acknowledgements}

The authors would like to thank the editor and the referees for their interesting comments, which greatly improved our paper. This work is supported by the National Natural Science Foundation of China (Grant No. 11661009), the Guangxi Science Fund for Distinguished Young Scholars (No. 2015GXNSFGA139001), and the Guangxi Natural Science Key Fund (No. 2017GXNSFDA198046). Innovation Project of Guangxi Graduate Education (No. YCSW2018046)

\section{Competing interests}

The authors declare to have no competing interests.

\section{Authors' contributions}

The work of Dr. GY is organizing and checking this paper, and Dr. WH mainly has done the experiments of the algorithms and written the codes. All authors read and approved the final manuscript.

\section{Publisher's Note}

Springer Nature remains neutral with regard to jurisdictional claims in published maps and institutional affiliations.

Received: 14 January 2018 Accepted: 26 April 2018 Published online: 11 May 2018

\section{References}

1. Birindelli, I., Leoni, F., Pacella, F.: Symmetry and spectral properties for viscosity solutions of fully nonlinear equations. J. Math. Pures Appl. 107(4), 409-428 (2017)

2. Ganji, D.D., Fakour, M., Vahabzadeh, A., et al.: Accuracy of VIM, HPM and ADM in solving nonlinear equations for the steady three-dimensional flow of a Walter's B fluid in vertical channel. Walailak J. Sci. Technol. 11(7), 203-204 (2014)

3. Georgiades, F: Nonlinear equations of motion of L-shaped beam structures. Eur. J. Mech. A, Solids 65, 91-122 (2017) 
4. Dai, Z., Wen, F.: Some improved sparse and stable portfolio optimization problems. Finance Res. Lett. (2018). https://doi.org/10.1016/j.frl.2018.02.026

5. Dai, Z., Li, D., Wen, F.: Worse-case conditional value-at-risk for asymmetrically distributed asset scenarios returns. J. Comput. Anal. Appl. 20, 237-251 (2016)

6. Dong, X., Liu, H., He, Y.: A self-adjusting conjugate gradient method with sufficient descent condition and conjugacy condition. J. Optim. Theory Appl. 165(1), 225-241 (2015)

7. Dong, X., Liu, H., He, Y., Yang, X.: A modified Hestenes-Stiefel conjugate gradient method with sufficient descent condition and conjugacy condition. J. Comput. Appl. Math. 281, 239-249 (2015)

8. Liu, Y.: Approximate solutions of fractional nonlinear equations using homotopy perturbation transformation method. Abstr. Appl. Anal. 2012(2), 374 (2014)

9. Chen, P.: Christoph Schwab, sparse-grid, reduced-basis Bayesian inversion, nonaffine-parametric nonlinear equations. J. Comput. Phys. 316(C), 470-503 (2016)

10. Shah, F.A., Noor, M.A.: Some numerical methods for solving nonlinear equations by using decomposition technique. Appl. Math. Comput. 251(C), 378-386 (2015)

11. Waziri, M., Aisha, H.A., Mamat, M.: A structured Broyden's-like method for solving systems of nonlinear equations. World Appl. Sci. J. 8(141), 7039-7046 (2014)

12. Wen, F., He, Z., Dai, Z., et al.: Characteristics of investors risk preference for stock markets. Econ. Comput. Econ. Cybern. Stud. Res $48,235-254$ (2014)

13. Fletcher, R., Reeves, C.M.: Function minimization by conjugate gradients. Comput. J. 7(2), 149-154 (1964)

14. Al-Baali, M., Narushima, Y., Yabe, H.: A family of three-term conjugate gradient methods with sufficient descent property for unconstrained optimization. Comput. Optim. Appl. 60(1), 89-110 (2015)

15. Egido, J.L., Lessing, J., Martin, V., et al.: On the solution of the Hartree-Fock-Bogoliubov equations by the conjugate gradient method. Nucl. Phys. A 594(1), 70-86 (2016)

16. Huang, C., Chen, C.: A boundary element-based inverse-problem in estimating transient boundary conditions with conjugate gradient method. Int. J. Numer. Methods Eng. 42(5), 943-965 (2015)

17. Huang, N., Ma, C.: The modified conjugate gradient methods for solving a class of generalized coupled Sylvester-transpose matrix equations. Comput. Math. Appl. 67(8), 1545-1558 (2014)

18. Mostafa, E.S.M.E.: A nonlinear conjugate gradient method for a special class of matrix optimization problems. J. Ind. Manag. Optim. 10(3), 883-903 (2014)

19. Albaali, M., Spedicato, E., Maggioni, F.: Broyden's quasi-Newton methods for a nonlinear system of equations and unconstrained optimization, a review and open problems. Optim. Methods Softw. 29(5), 937-954 (2014)

20. Fang, X. Ni, Q.. Zeng, M.: A modified quasi-Newton method for nonlinear equations. J. Comput. Appl. Math. 328, 44-58 (2018)

21. Luo, Y.Z., Tang, G.J., Zhou, L.N.: Hybrid approach for solving systems of nonlinear equations using chaos optimization and quasi-Newton method. Appl. Soft Comput. 8(2), 1068-1073 (2008)

22. Tarzanagh, D.A., Nazari, P., Peyghami, M.R.: A nonmonotone PRP conjugate gradient method for solving square and under-determined systems of equations. Comput. Math. Appl. 73(2), 339-354 (2017)

23. Wan, Z., Hu, C., Yang, Z: A spectral PRP conjugate gradient methods for nonconvex optimization problem based on modified line search. Discrete Contin. Dyn. Syst., Ser. B 16(4), 1157-1169 (2017)

24. Yuan, G., Sheng, Z., Wang, B., et al.: The global convergence of a modified BFGS method for nonconvex functions. J. Comput. Appl. Math. 327, 274-294 (2018)

25. Amini, K., Shiker, M.A.K., Kimiaei, M.: A line search trust-region algorithm with nonmonotone adaptive radius for a system of nonlinear equations. 4OR 14,133-152 (2016)

26. Qi, L., Tong, X.J., Li, D.H.: Active-set projected trust-region algorithm for box-constrained nonsmooth equations. J. Optim. Theory Appl. 120(3), 601-625 (2004)

27. Yang, Z., Sun, W., Qi, L.: Global convergence of a filter-trust-region algorithm for solving nonsmooth equations. Int. J. Comput. Math. 87(4), 788-796 (2010)

28. Yu, G.: A derivative-free method for solving large-scale nonlinear systems of equations. J. Ind. Manag. Optim. 6(1), 149-160 (2017)

29. Li, Q., Li, D.H.: A class of derivative-free methods for large-scale nonlinear monotone equations. IMA J. Numer. Anal. 31(4), 1625-1635 (2011)

30. Sheng, Z., Yuan, G., Cui, Z:: A new adaptive trust region algorithm for optimization problems. Acta Math. Sci. 38B(2), 479-496 (2018)

31. Sheng, Z., Yuan, G., Cui, Z., et al.: An adaptive trust region algorithm for large-residual nonsmooth least squares problems. J. Ind. Manag. Optim. 34, 707-718 (2018)

32. Yuan, G., Sheng, Z., Liu, W.: The modified HZ conjugate gradient algorithm for large-scale nonsmooth optimization PLOS ONE 11, 1-15 (2016)

33. Yuan, G. Sheng, Z: Nonsmooth Optimization Algorithms. Press of Science, Beijing (2017)

34. Narushima, Y., Yabe, H., Ford, J.A.: A three-term conjugate gradient method with sufficient descent property for unconstrained optimization. SIAM J. Optim. 21(1), 212-230 (2016)

35. Zhou, W.: Some descent three-term conjugate gradient methods and their global convergence. Optim. Methods Softw. 22(4), 697-711 (2007)

36. Cardenas, S.: Efficient generalized conjugate gradient algorithms. I. Theory. J. Optim. Theory Appl. 69(1), 129-137 (1991)

37. Dai, Y.H., Yuan, Y.: A nonlinear conjugate gradient method with a strong global convergence property. SIAM J. Optim. 10(1), 177-182 (1999)

38. Hestenes, M.R., Steifel, E.: Cassettari, D., et al.: Method of conjugate gradients for solving linear systems. J. Res. Natl. Bur. Stand. 49(6), 409-436 (1952)

39. Wei, Z., Yao, S., Liu, L.: The convergence properties of some new conjugate gradient methods. Appl. Math. Comput. 183(2), 1341-1350 (2006)

40. Yuan, G., Lu, X:: A modified PRP conjugate gradient method. Ann. Oper. Res. 166(1), 73-90 (2009)

41. Yuan, G. Lu, X., Wei, Z:: A conjugate gradient method with descent direction for unconstrained optimization. J. Comput. Appl. Math. 233(2), 519-530 (2009) 
42. Yuan, G., Meng, Z., Li, Y.: A modified Hestenes and Stiefel conjugate gradient algorithm for large-scale nonsmooth minimizations and nonlinear equations. J. Optim. Theory Appl. 168(1), 129-152 (2016)

43. Yuan, G., Wei, Z., Lu, X.: Global convergence of BFGS and PRP methods under a modified weak Wolfe-Powell line search. Appl. Math. Model. 47, 811-825 (2017)

44. Zhang, L., Zhou, W., Li, D.H.: A descent modified Polak-Ribière-Polyak conjugate gradient method and its global convergence. IMA J. Numer. Anal. 26(4), 629-640 (2006)

45. Nazareth, L.: A conjugate direction algorithm without line searches. J. Optim. Theory Appl. 23(3), 373-387 (1977)

46. Yuan, G., Wei, Z., Li, G.: A modified Polak-Ribière-Polyak conjugate gradient algorithm for nonsmooth convex programs. J. Comput. Appl. Math. 255, 86-96 (2014)

47. Yuan, G., Zhang, M.: A three-terms Polak-Ribière-Polyak conjugate gradient algorithm for large-scale nonlinear equations. J. Comput. Appl. Math. 286, 186-195 (2015)

48. Andrei, N.: An unconstrained optimization test functions collection. Environ. Sci. Technol. 10(1), 6552-6558 (2008)

49. Dolan, E.D., Moré, J.J.: Benchmarking optimization software with performance profiles. Math. Program. 91(2), 201-213 (2001)

50. Ahmad, F., Tohidi, E., Ullah, M.Z., et al.: Higher order multi-step Jarratt-like method for solving systems of nonlinear equations, application to PDEs and ODEs. Comput. Math. Appl. 70(4), 624-636 (2015)

51. Kang, S.M., Nazeer, W., Tanveer, M., et al.: Improvements in Newton-Raphson method for nonlinear equations using modified Adomian decomposition method. Int. J. Math. Anal. 9(39), 1910-1928 (2015)

52. Matinfar, M., Aminzadeh, M.: Three-step iterative methods with eighth-order convergence for solving nonlinear equations. J. Comput. Appl. Math. 225(1), 105-112 (2016)

53. Papp, Z., Rapajić, S.: FR type methods for systems of large-scale nonlinear monotone equations. Appl. Math. Comput. 269(C), 816-823 (2015)

54. Yuan, G., Wei, Z., Lu, X.: A new backtracking inexact BFGS method for symmetric nonlinear equations. Comput. Math. Appl. 55(1), 116-129 (2008)

55. Li, Q., Li, D.H.: A class of derivative-free methods for large-scale nonlinear monotone equations. IMA J. Numer. Anal. 31(4), 1625-1635 (2011)

56. Solodov, M.V., Svaiter, B.F:: A hybrid projection-proximal point algorithm. J. Convex Anal. 6, $59-70$ (1999)

57. Ceng, L.C., Wen, C.F., Yao, Y.: Relaxed extragradient-like methods for systems of generalized equilibria with constraints of mixed equilibria, minimization and fixed point problems. J. Nonlinear Var. Anal. 1, 367-390 (2017)

58. Cho, S.Y.: Generalized mixed equilibrium and fixed point problems in a Banach space. J. Nonlinear Sci. Appl. 9, 1083-1092 (2016)

59. Cho, S.Y.: Strong convergence analysis of a hybrid algorithm for nonlinear operators in a Banach space. J. Appl. Anal. Comput. 8, 19-31 (2018)

60. Liu, Y:: A modified hybrid method for solving variational inequality problems in Banach spaces. J. Nonlinear Funct. Anal. 2017, Article ID 31 (2017)

61. Solodov, M.V., Svaiter, B.F.: A Globally Convergent Inexact Newton Method for Systems of Monotone Equations, Reformulation, Nonsmooth, Piecewise Smooth, Semismooth and Smoothing Methods, pp. 1411-1414. Springer, Berlin (1998)

\section{Submit your manuscript to a SpringerOpen ${ }^{\circ}$ journal and benefit from:}

- Convenient online submission

- Rigorous peer review

Open access: articles freely available online

- High visibility within the field

- Retaining the copyright to your article

Submit your next manuscript at $\gg$ springeropen.com 